\title{
Development and Application of Database System for Rubber Material
}

\author{
Ningfei $\mathrm{Wu}^{1, \mathrm{a}^{*}}$, Yan $\mathrm{Cao}^{2, \mathrm{~b}}, \mathrm{Yu} \mathrm{Bai}^{3, \mathrm{c}}$ and Hu Qiao ${ }^{4, \mathrm{~d}}$
}

Department of Mechanical and Electronic Engineering, Xi'an Technological University, Xi'an, China 835847187@qq.com, jantonyz@163.com, baiyv@xatu.edu.cn, qiaonwpu@hotmail.com

Keywords: Rubber; Database; Inquiring

\begin{abstract}
In order to quickly and comprehensively understand some rubber material data, the three layer system structure based on B_S model develop database system of rubber materials by using ASP technology, the Windows7 operating system, IIS7.5 as Web server, Dreamweaver CS6 as the development platform, SQL Server2008 as the backstage database, and introduces the database system structure design and five function module. The results show that the system classifies the scientific management of rubber material data, provide a variety of efficient query methods and user management means, realize the effective maintenance of the rubber material database system. The system has a friendly interface, easy to use, easy to understand, complete functions, greatly improving user query efficiency.
\end{abstract}

\section{Introduction}

It is one of the important materials in the chemical industry for rubber to widely use in manufacture of tires, rubber belt, and rubber shoes and materials parts. It is said that manufacture of rubber tires is the largest consumption market. ${ }^{[1]}$ In recent years, with the rapid development of rubber industry, information of the rubber grade, type, property, technology and structure has reached an amazing number. If we want to know some data of rubber material and relevant knowledge by traditional way that people inquiry a large number standards manuals, literature data and charts, It is a heavy task for Engineer and will waste a lot of time. Therefore, the use of the database system has become a very realistic and urgent problem to classify and apply a large number of complicated data information.

Many developed countries in the world have launched the work of material database and research much the system software. Especially the United States, Japan and some European countries, they have all kinds of database. The integrated network business database-Mat Web in American is known as its universal and convenient. Japan Science and Technology Corporation developed solid diffusion database network based on internet support research and material design, including iron, nickel and tungsten alloy. SOLMA, metal database, established The German technology testing association contains kinds of ferrous and non-ferrous metals that its types reached more than 3000 species and its data reached more than $20000 .{ }^{[2]}$

The research of materials database began in the late 1980s in china, so far the various walks of life, in various fields of scientific research and production agencies have established a large number of different the material database. For example, the mechanical engineering material database established Shanghai Material Research Institute has completed property comprehensive test of 180 steel and obtained more than 200 thousand data. The Science database established China Academy is the largest amount of information at home professional service, most widely, the highest level, the most comprehensive scientific information service system. ${ }^{[3]}$

\section{Database System Design}

\section{Development Platforms and Tools.}

System development environment: Dreamweaver CS6

System development language: ASP

System background database: SQL Server 2008

Operating system: Windows 7 
Web server: IIS 7.5

Browser: IE or other browsers

Database Structure Design.

Conceptual Structure Design. Conceptual structure design is the abstraction of user demand as the information structure. The tool that describes the conceptual structure is the E-R model. This is a reference to CHINA AERONAUTICAL MATERIALS HANDBOOK and the manual collection of rubber materials can be divided into 15 species and including 466 material grades, 204 material standards and 76 material names. Each category contains a large number of data, tables and graphics, so the information of the rubber material is various and complex.

The paper is based on scientific classification of material information, using relational database to design the structure and function of the system. The rubber material database include: material information, application situation, aging resistance, dielectric properties, process, material standard, use recommendations and other information. The material information contains material, material name. Material type, basic composition, supply condition and production unit. Application material contains application scope and main purposes. The using technology contains material, processing method, curing methods and precautions. Aging properties contains material, aging time, and aging temperature. The dielectric properties contain material, medium temperature, time, material. The standard includes material and material standards. Suggestions contain material grades and recommendations. Others contain material, packaging, transportation and storage of oak. The specific E-R model of the adhesive material database is shown in Fig 1.

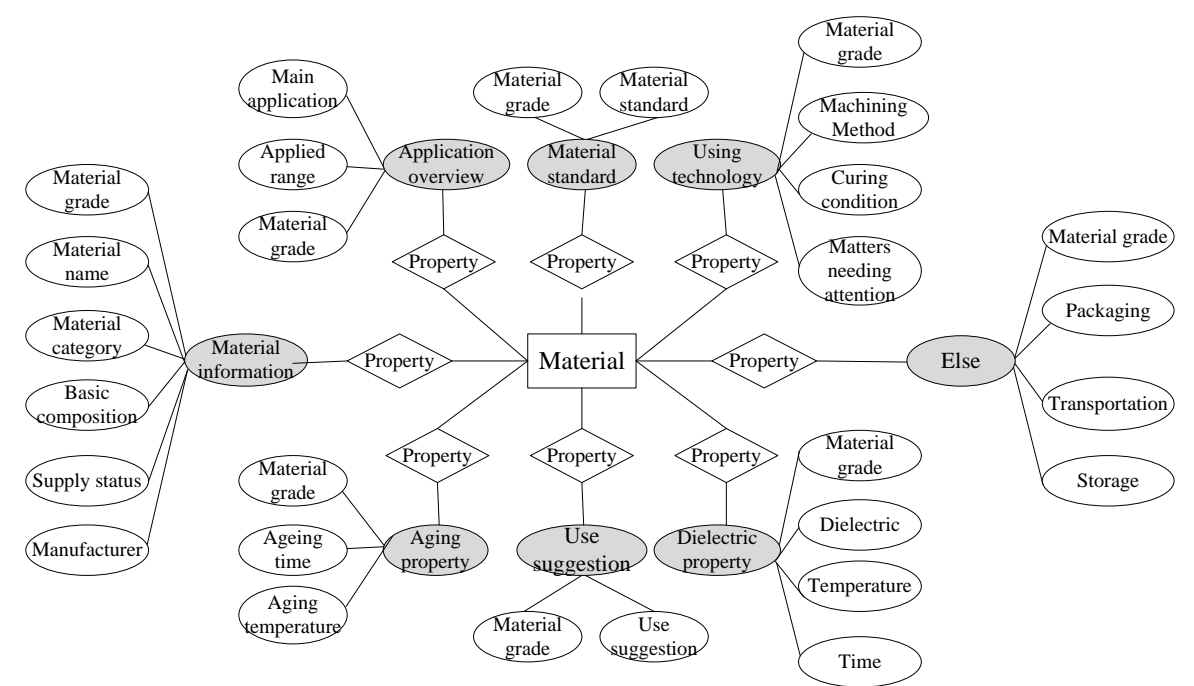

Figure 1. Database structure of rubber material

Logical Structure Design. The logical structure design is to determine the data model, which is characterized by high data independence, low redundancy, data consistency and integrity. [4]Relational model database system is chosen in this paper, which is made up by some data tables that are established according to the standard of three normal form and table contrast to a relation in the database system. According to the E-R model, the transformation of each entity see table 1 .

Table 1 Material information sheet

\begin{tabular}{ccccc}
\hline FIELD NAME & TYPE & SIZE & NOT NULL & EXPLIAN \\
\hline MG & CHAR & 10 & NOT NULL & PK \\
MN & CHAR & 20 & NOT NULL & \\
MC & CHAR & 50 & NOT NULL & \\
BC & VARCHAR & 50 & NULL & \\
MS & VARCHAR & 100 & NULL & \\
MM & VARCHAR & 100 & NULL & \\
\hline
\end{tabular}




\section{System Function Module Design}

The database system puts to use the idea of structured system analysis and design, a top-down division style divide system reasonably, the complex system is decomposed into relatively independent, single function module.[5] The database system is divided into the rubber material, data management, system maintenance, user management and system help module. The 5 function modules of the system are shown in Fig 2.

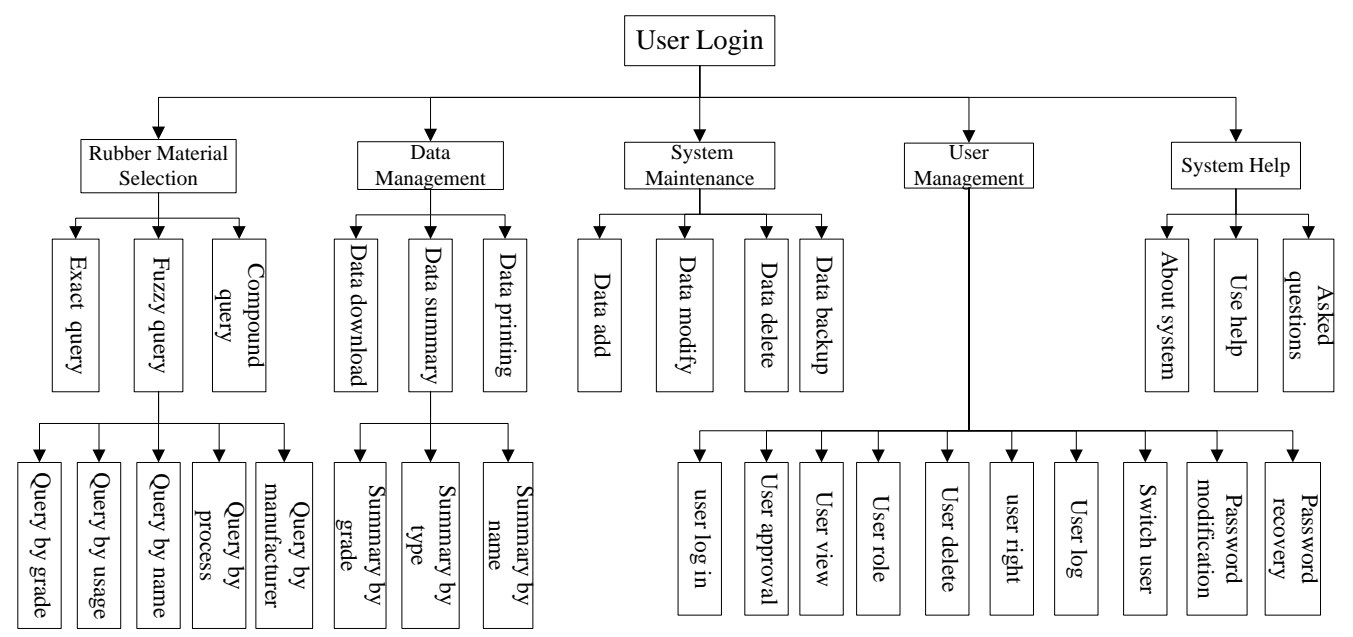

Figure. 2. Functional modules of the system

Login Interface. Fig. 3 is a login interface of database system of rubber material. The corresponding input username and password in the login interface and click "login" button to automatically enter the database system of rubber materials after a few seconds later.
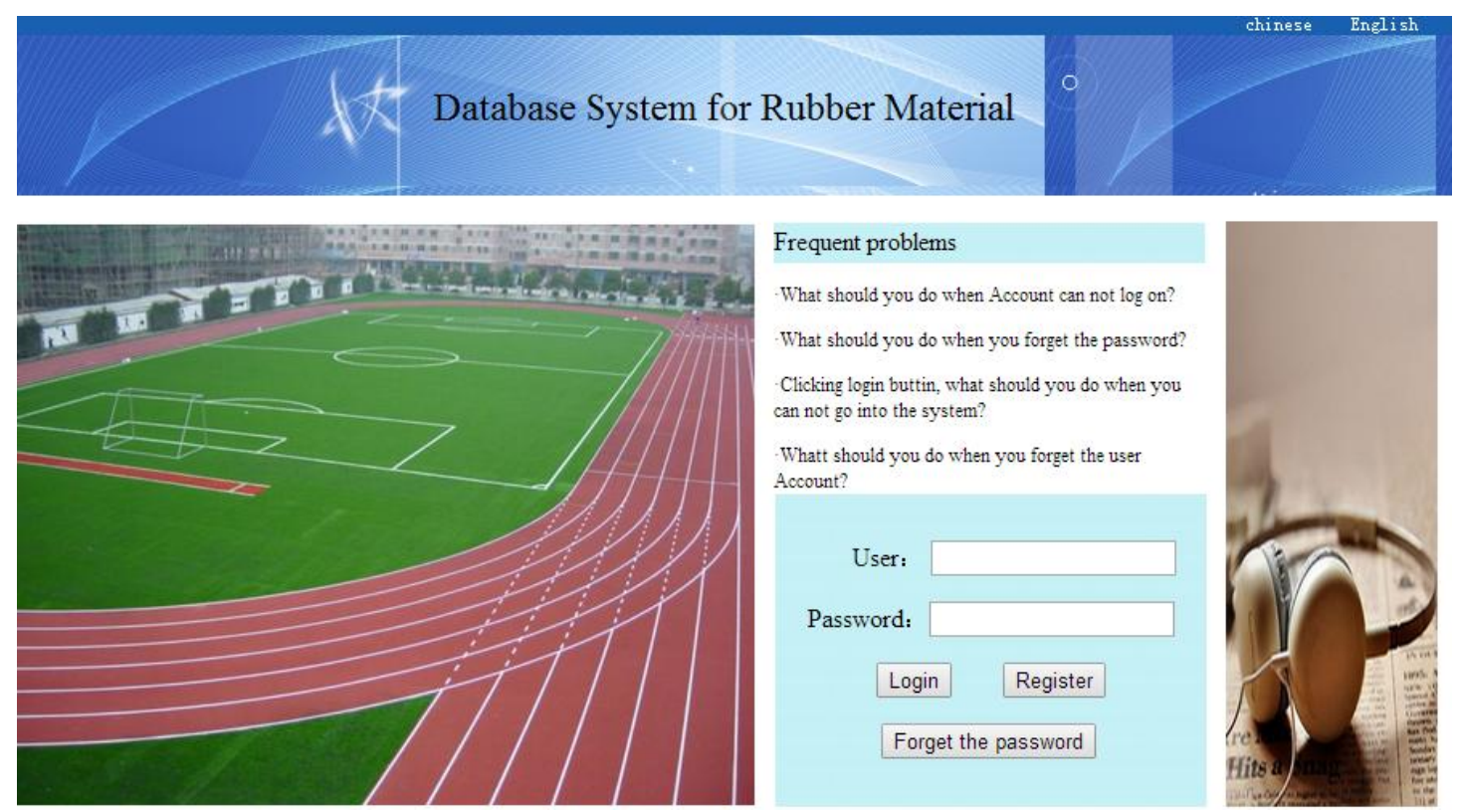

Figure 3. The of interface system login

Rubber Material Selection Function Module. The rubber material module contains totally third inquiry: fuzzy query, compound query exact query. Fuzzy query can also be divided into query by grade, query by usage, query by process and query by manufacturer. Here only introduce the query by grade of fuzzy query mode. Click on the "query by grade ", then input the query grades 
on the right in the text box and click "search" button, finally the browser will display the search results. The results are shown in Fig. 4.

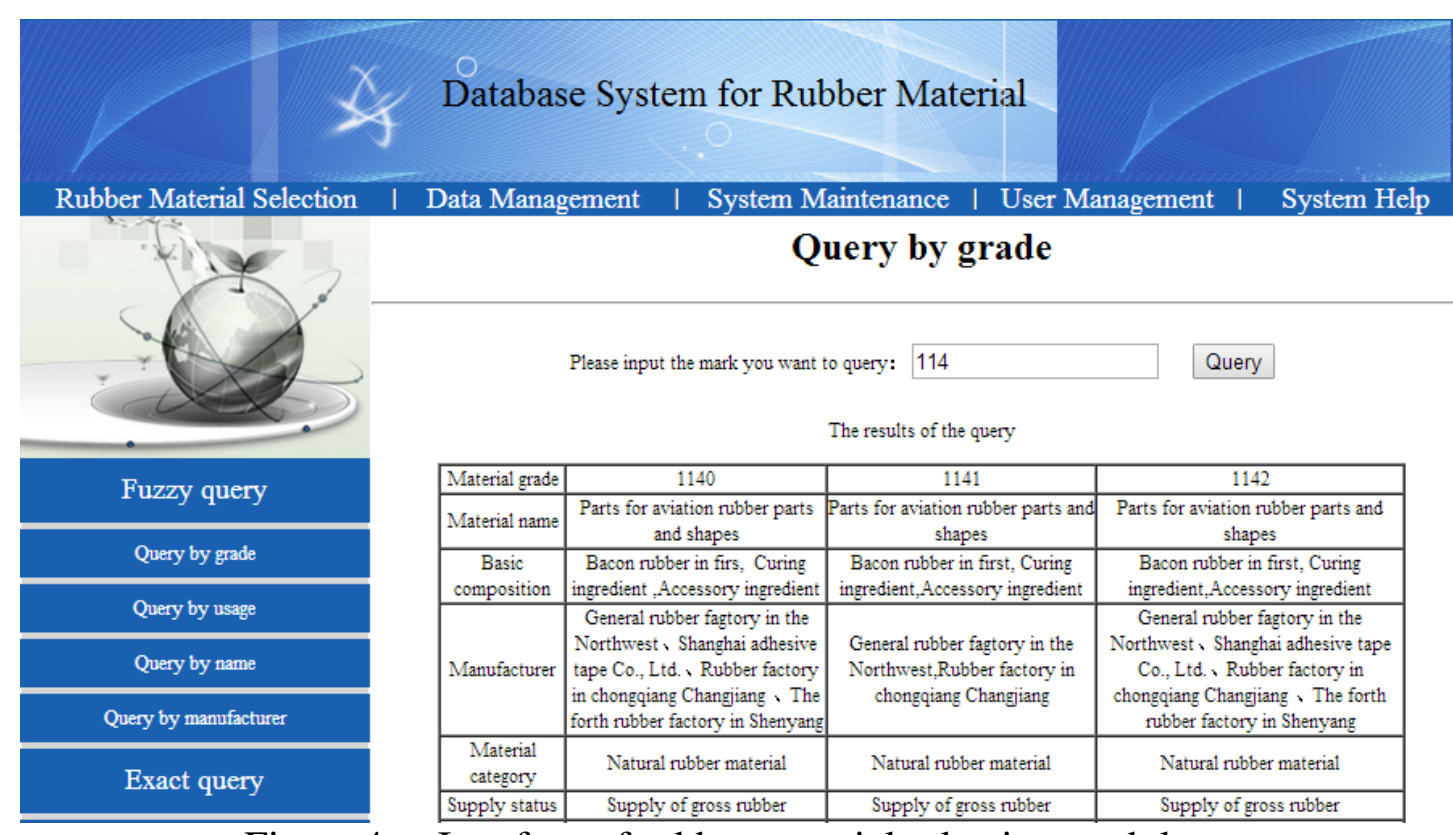

Figure 4. Interface of rubber material selection module

System Maintenance Function Module. System maintenance function module is an important field of system data that constantly enriched and improved. The system administrator can add, delete, modify and backup data to material database through the function module and realize the management and maintenance of the database. [6]

It is system administrator that can operate the module. First of all, clicking the system maintenance and the system will display the system administrator login interface. After enter the user and password, he or she can enter the system maintenance interface and handle with the rubber material data. Here only introduce the data add function that is shown in Fig. 5.

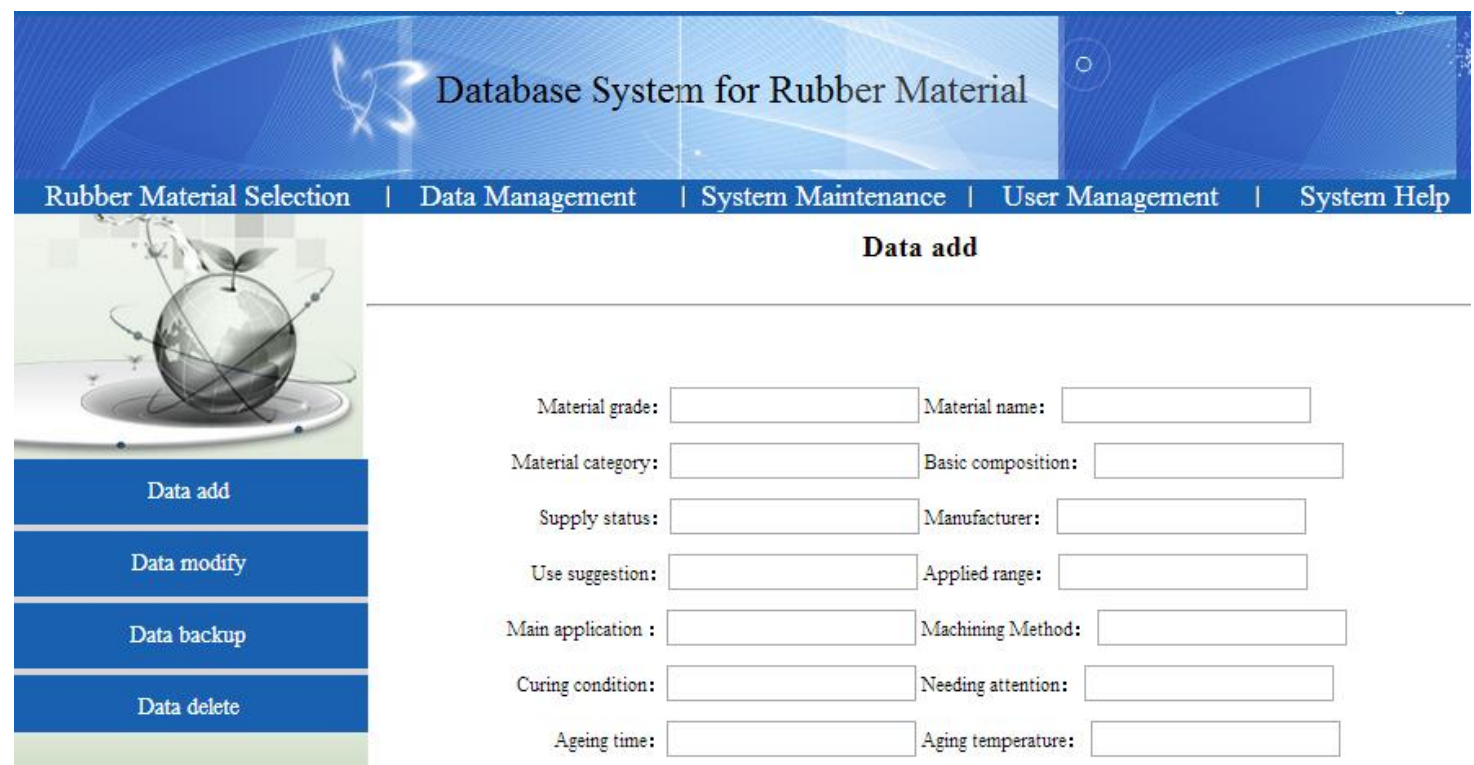

Figure 5. Interface of system maintenance module

User Management Function Module. The user management is to make sure the security of data. It can allocate the username, password and access permissions. Permissions include ordinary users and administrators. Ordinary users can only use the data query, data input and output functions in 
the system, but he or she can't change the contents of the database; the administrator can not only manage and maintain the materials database, but also be assigned a username and password.

Click on the menu user management system, the system will show the corresponding functions: user log in, user approval, user view, user roles, users delete, user permissions, user log, user switching, modify password and password function. The user interface is shown in Fig. 6.

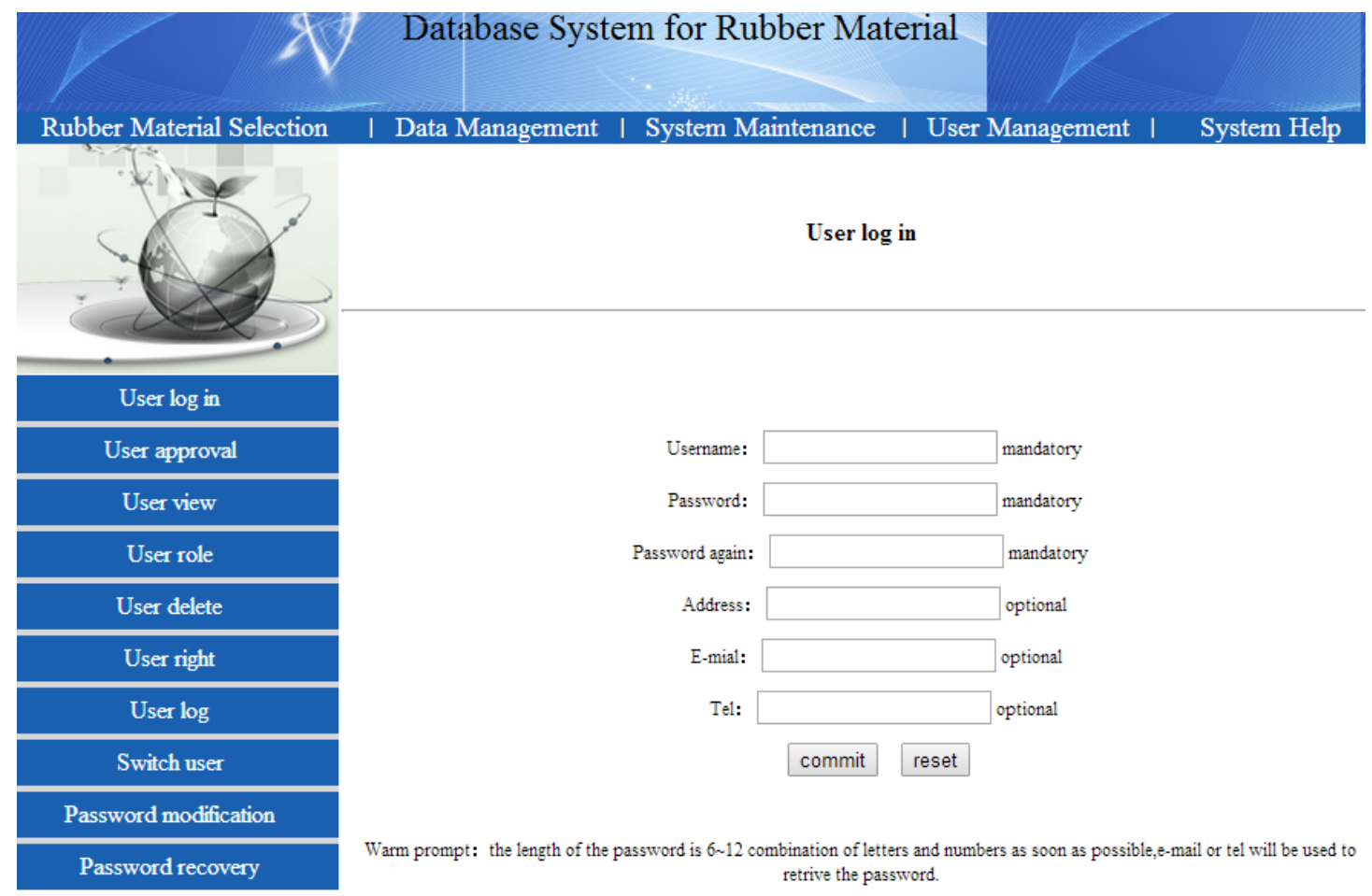

Figure 6. Interface of user management module

\section{Summary}

In the development of database system keeping to the practical, advanced, extension principle, the paper establish a convenient database system that can select material quickly and manage scientifically category, grade, main uses, database system manufacturers, use technology and other material information of rubber materials. The system interface is friendly, simple operation, intuitive and accurate query, practical strong, the application of this system is helpful for manufacturing workers to select rubber material reasonably. Under the premise of ensuring the rubber life, it is more impossible to reduce the rubber material cost.

\section{Acknowledgment}

The paper is supported by Key Problem Tackling Project of Shaanxi Scientific and Technological Office (2016GY-024) and non-traditional machining Key Laboratory Project of Shaanxi Province (15JS041).

\section{References}

[1] Xia Li. Hang Su. Gang Xie. Xiaoling Chen. Caifu Yang. Present Status and Future of Materials Database China Metallurgy [J].2007.

[2] Lei Chen. Tendency and Problems of Our National Database [J].Modern Intelligence, 2006.

[3] Lei Zhang. Research and Development of Wrought Magnesium Alloy Data Development Platfrom[J]. Chongqing University, 2015. 
[4] Xuedong Wang. Database Management System of Welding Material [J]. Journal of Shenyang University of Technology, 2001.

[5] HE Tao. Research on the Development of Material Database and Technological Standard Query System for Plastic Mould [D].Wuhan University Of Technology, 2011.

[6] Ai-lin Zhou. Development of Mechanical Processing Materials Database System [D].Xi'An Technology University, 2013.

[7] Xiji Su. The Development and Application of Database System for Plastic Mould Material Based on C/S [D].Jiang Su University, 2007.

[8] Xinrui Zhang. The Design and Implementation of Student Information Management System Based On ASP Technology [D]. Tianjin Normal University, 2012.

[9] Zhiqiang Yuan. Design and Development on Magnesium Alloys Database Based on Web [D] Chongqing University, 2006.

[10]Lei Yang. Design and Implementation of Shopping System Based on ASP Technology [D]. Xiamen University, 2013. 